\title{
Editorial
}

\section{Applications of Fiber Reinforced Polymer Composites}

\author{
Togay Ozbakkaloglu, ${ }^{1}$ Jian-Fei Chen, ${ }^{2}$ Scott T. Smith, ${ }^{3}$ and Jian-Guo Dai ${ }^{4}$ \\ ${ }^{1}$ University of Adelaide, Adelaide, SA 5005, Australia \\ ${ }^{2}$ Queen's University Belfast, Belfast BT7 1NN, UK \\ ${ }^{3}$ Southern Cross University, Lismore, NSW 2480, Australia \\ ${ }^{4}$ Hong Kong Polytechnic University, Kowloon, Hong Kong \\ Correspondence should be addressed to Togay Ozbakkaloglu; togay.ozbakkaloglu@adelaide.edu.au
}

Received 19 January 2016; Accepted 20 January 2016

Copyright ( $) 2016$ Togay Ozbakkaloglu et al. This is an open access article distributed under the Creative Commons Attribution License, which permits unrestricted use, distribution, and reproduction in any medium, provided the original work is properly cited.

Fueled by the need to surpass the limitations of conventional materials, recent years have seen a large increase in engineering applications of advanced fiber reinforced polymer (FRP) composite materials in many major industries, such as aerospace and defense, automotive, construction, marine, and oil and gas. FRP composites are very attractive for these applications due to their highly favorable material properties, including high strength-to-weight and stiffness-to-weight ratios, and corrosion resistance. Studies conducted to date have demonstrated the numerous advantages offered by FRP composites in various engineering applications. However, there are still a number of technical and implementation issues that need to be resolved prior to broader uptake of the application of FRP composites by some engineering communities such as civil construction. This special issue is aimed at disseminating the most recent advances and developments in this exciting field.

A total of 17 papers were submitted to the special issue and after a rigorous peer-review process six of them were accepted to appear in the issue as original research articles. These six papers deal with a range of topics on the structural behavior of composite members/structures and mechanical properties and development of composite materials. The studies on the former investigate the dynamic behavior of composite FRP bridge systems and flexural behavior of previously damaged steel beams repaired with FRP. The studies on the latter are concerned with the development of ecoefficient engineered cementitious composites, mechanical properties of carbon fiber composites obtained using different molding techniques, and properties of high-density and ultra-high molecular weight polyethylene and heat-treated bamboo fiber composites.

We hope that some of the most recent advances on the development and applications of FRP composites that have been disseminated in this special issue will be of interest to readers and contribute toward the advancement of research in the field.

\section{Acknowledgments}

We would like to thank all the authors for their contributions and reviewers for their valuable comments and suggestions.

\author{
Togay Ozbakkaloglu \\ Jian-Fei Chen \\ Scott T. Smith \\ Jian-Guo Dai
}



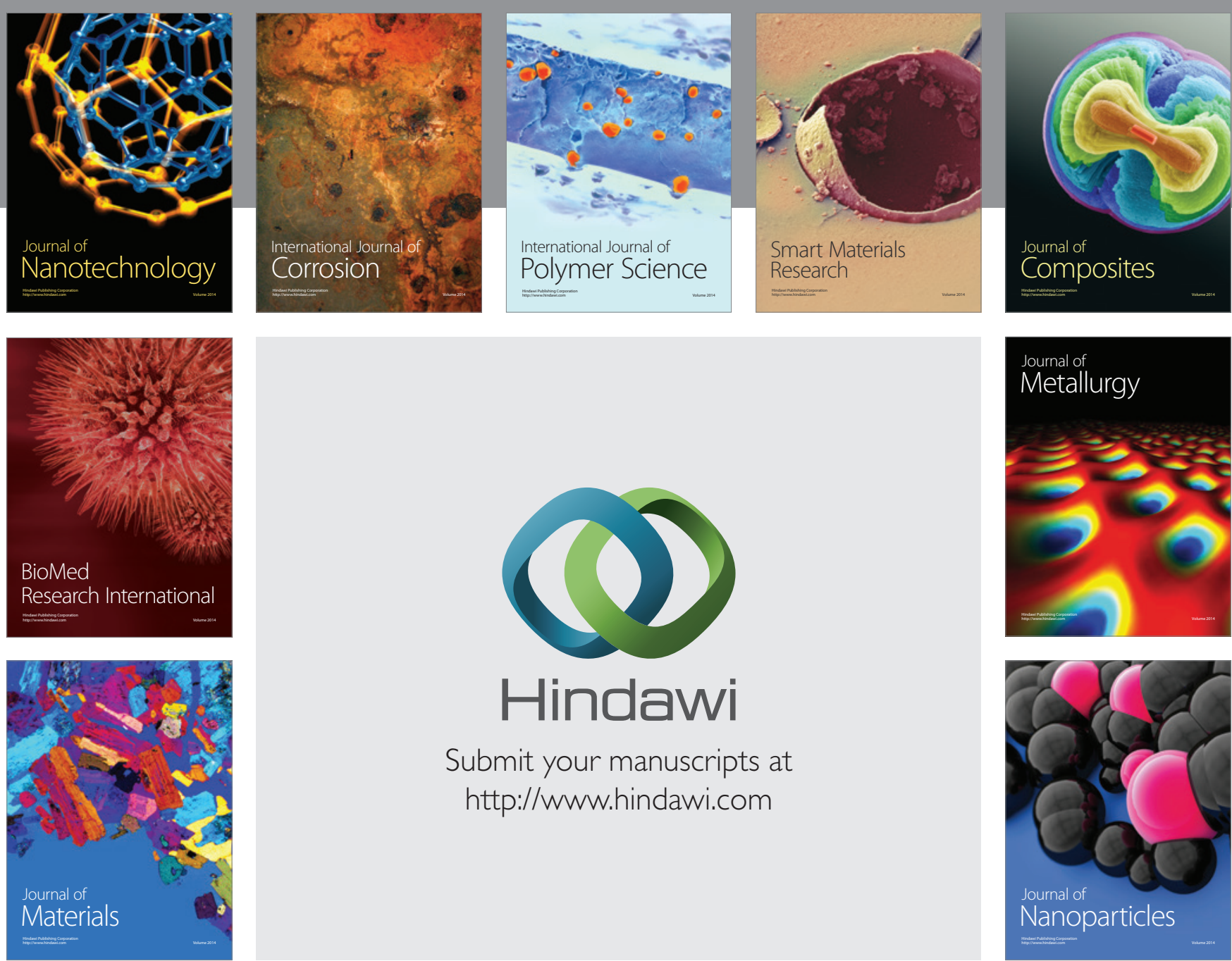

\section{Hindawi}

Submit your manuscripts at

http://www.hindawi.com

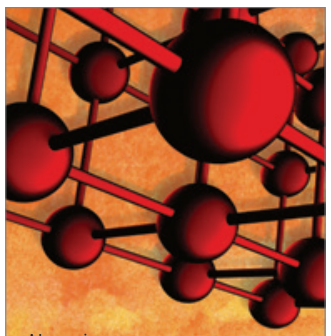

Materials Science and Engineering
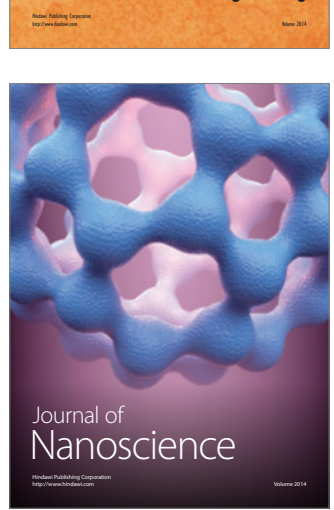
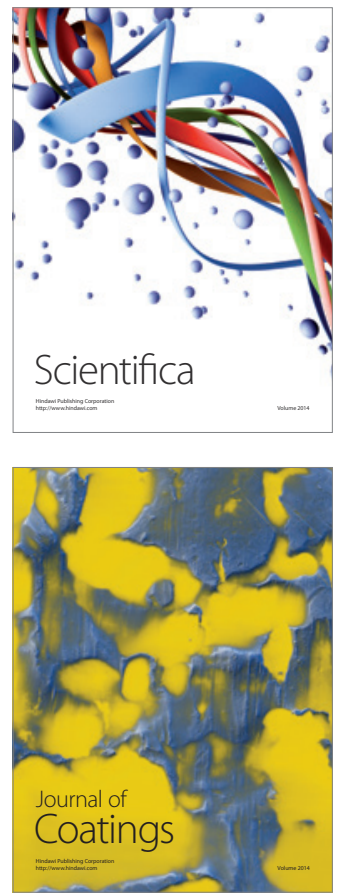
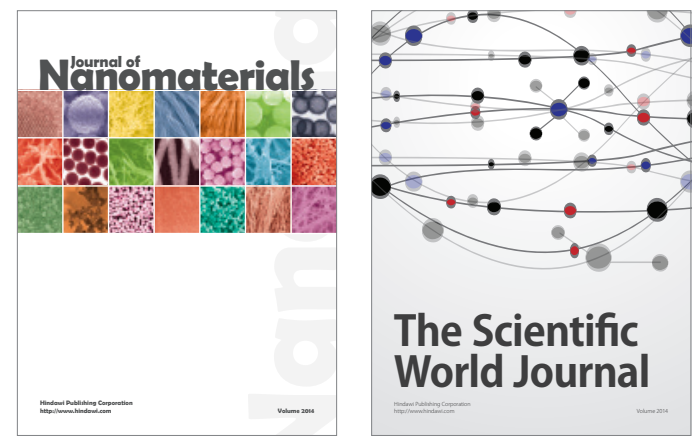

The Scientific World Journal
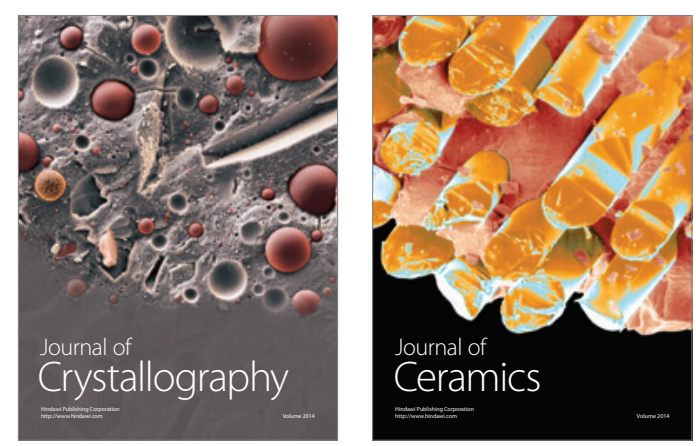
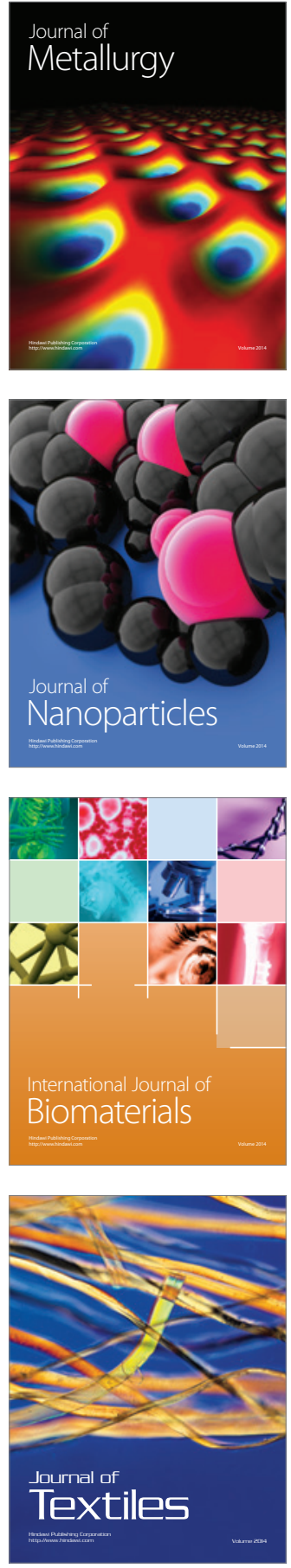